\title{
A reexamination of duplex perception evoked by intensity differences
}

\author{
PETER J. BAILEY and PAUL HERRMANN \\ University of York, York, England
}

\begin{abstract}
Duplex perception is a term used to describe the simultaneous evocation of phonetic and nonphonetic percepts, a phenomenon held by some to reveal the operation of a specialized module for phonetic perception. In a widely cited experiment by Whalen and Liberman (1987), duplex percepts were created by replacing the third formant transition in a synthetic syllable with a sinusoidal tone glide and manipulating the level of the glide relative to the rest of the syllable. We discuss this study, and report four experiments. The first two made a systematic estimate of the "duplexity threshold"- the tone glide level at which a chirp-like sound could be heard as well as the speech-like syllable. In the third and fourth experiments, we used the results of the first to repeat and extend the experimental procedures in the original Whalen and Liberman study. We discuss the differences in outcome, and conclude that our results may be explained without the need to invoke a specialized phonetic module.
\end{abstract}

When certain elements of a speech-like sound are made more intense than or spatially dislocated from the rest, both phonetic and nonphonetic percepts are evoked simultaneously. This phenomenon, originally reported by Rand (1974), has been called duplex perception. It continues to attract attention, both from those who wish to affirm that it indicates something special about phonetic perception and from those who believe it does not. This paper is concerned with an attempt to repeat and extend a widely cited experiment on duplex perception by Whalen and Liberman (1987).

There are several experimental methods used to demonstrate duplex percepts. In the most commonly reported procedure, a synthetic consonant-vowel syllable is presented to a listener such that the syllable "base"-all of the syllable apart from the initial third formant frequency transition-is played to one ear while the third formant transition is played (in its correct temporal alignment) to the other ear. If the pattern of formant transitions is arranged so that the direction of the third formant transition is the distinctive property that determines the identity of the initial consonant, as seen in Figure 1 , it is readily shown that the third formant transition can exert its phonetically contrastive influence, even when presented dichotically with the base. Thus, in the example of Figure 1 , the subject will report either "da" or "ga" according to whether the third formant transition is falling or rising. However, in addition to reporting a coherent phonetic percept from the ear to which the base is presented, listeners also typically report hearing simulta-

The support of the U.K. Science and Engineering Research Council and Medical Research Council is acknowledged. We are very grateful for comments received from Valter Ciocca, Alvin Liberman, Dick Pastore, Brian Roberts, Doug Whalen, and an anonymous reviewer. Address correspondence to P. J. Bailey, Department of Psychology, University of York, York YO1 5DD, England. neously a nonphonetic chirp-like sound in the ear to which the isolated transition is presented. This "chirp" is similar to the percept evoked when the transition is presented alone without the base.

The simultaneous experience of phonetic and nonphonetic acoustic events in duplex perception has been taken as support for the notion that phonetic events and other nonspeech acoustic events are perceived by distinct processes (e.g., Mann \& Liberman, 1983). In a more explicit version of this view (e.g., Liberman \& Mattingly, 1989), a special phonetic module has direct (and preferential) access to the primary auditory representation, from which it extracts phonetically relevant information, leaving an auditory residue for access by processes that determine the conventional auditory dimensions of loudness, pitch, and timbre.

This proposal has not gone unchallenged. It has been claimed that the duplex perception result could be explained without resort to distinct perceptual modules if subjects can identify the consonant on the basis of information from the chirp alone (Nusbaum, 1984; Nusbaum, Schwab, \& Sawusch, 1983; but see also Repp, 1984). Another counterargument has drawn attention to the existence of duplex-like perceptual phenomena for nonspeech sounds (Fowler \& Rosenblum, 1990; Pastore, Schmuckler, Rosenblum, \& Szczesiul, 1983) and for nonauditory modalities (Bregman, 1987). If the demonstration of duplex perception with a particular stimulus ensemble necessarily implies the existence of a specialized perceptual module, these diverse demonstrations of duplex perception imply a proliferation of specialized perceptual modules. Other experiments have demonstrated an interdependence of auditory and phonetic perceptual processes that is inconsistent with the special, preemptive status of the proposed phonetic module (e.g., Ciocca \& Bregman, 1989). 


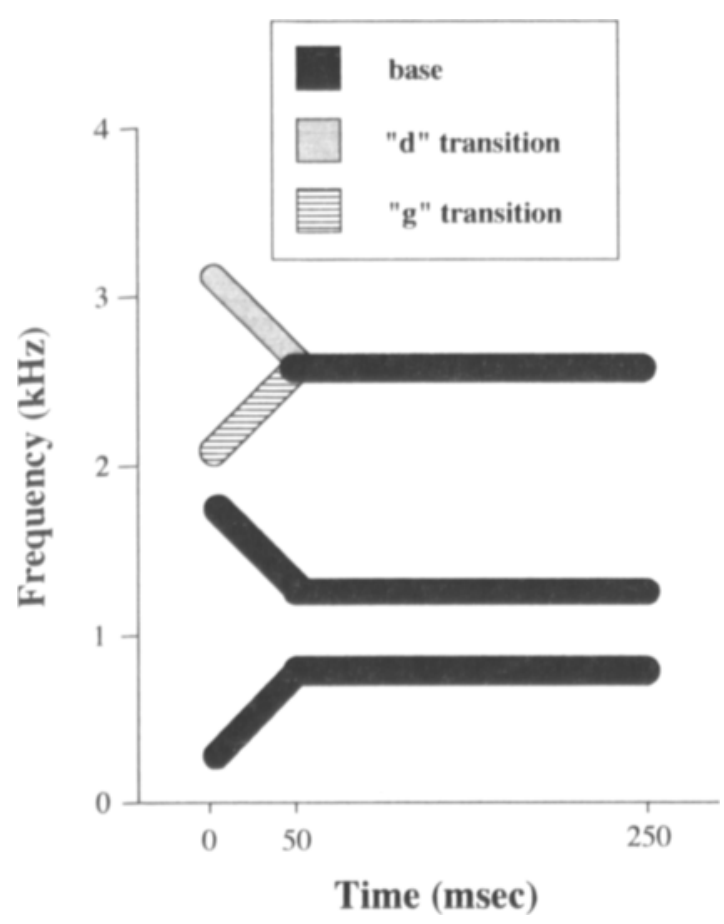

Figure 1. Schematic formant patterns sufficient for synthesis of "da" and "ga."

Whalen and Liberman (1987) used a variant of the procedure described above, in which the third formant transition was replaced by a sinusoidal tone glide with frequency characteristics similar to those of the formant transition. The duplex percept was manipulated by varying the level of the tone glide relative to that of the third formant steady state. Furthermore, their procedure did not require dichotic separation of the base and transition, as both components of the syllable were presented binaurally. The attraction of these modifications lay in the expectations that (1) sinusoidal tone glides would be more discriminable and less likely than a formant transition to provide information in isolation about consonant identity, and (2) that binaural presentation would be more likely to lead to the perceptual integration of base and transition.

To establish that this modified procedure led to bona fide duplex perception consistent with the modularity of phonetic processing, Whalen and Liberman (1987) sought to show that (1) in isolation, the falling and rising tone glides cannot reliably be identified with " $d$ " and "g," (2) there is a glide intensity-the "duplexity threshold"that will be just sufficient to evoke nonspeech "chirp" percepts when base and glide are presented together, (3) above the duplexity threshold, the chirps in syllabic context can be matched to those evoked by glides presented in isolation, and (4) above and below the duplexity threshold, the direction of the glide in syllabic context determines whether the syllable is heard as "da" or "ga."

Accordingly, Whalen and Liberman's (1987) experiment began with a condition that sought to show that falling and rising glides in isolation were not labeled reliably as " $d$ "'-like and " $g$ "'-like, respectively. Their subjects were asked to identify as "da" or "ga" a randomized sequence of isolated rising and falling tone glides incorporating 20 of each type. Taken together, the identification performance of the 10 subjects $(64.8 \%$ correct) was not significantly better than chance. However, it is clear that, for some of the listeners, falling and rising tone glides were identified reliably with " $d$ " and " $g$ " responses, respectively. Evidently, in an experiment like this involving only two stimuli and two responses, if subjects can discriminate the stimuli and make an initial arbitrary assignment of responses to stimuli that they hold to consistently, such "correct" responses are inevitable. Nonetheless, there remains the possibility that the initial assignment of responses to stimuli is not completely arbitrary for some subjects, but is governed by the presence in isolated rising and falling glides of some perceptual properties in common with " $d$ " and " $g$ " percepts. The possibility that such schematic but distinctive phonetic information is potentially available is strengthened by the fact that, at $65 \%$ correct, performance deviates from chance $(50 \%)$ in the direction to be expected if some phonetic information is indeed available in the glides. The use of such phonetic information, if it is present, is not convincingly ruled out by Whalen and Liberman's data.

We were also concerned by a second issue related to this. Even if glide identification performance is at chance at the start of the experiment, exposure to the tone glides throughout the experiment may lead at some point to the adoption of a systematic and correct response strategy, even in the absence of feedback about the correctness of responses in later experimental conditions. This sort of spontaneous change in the perception of tonal signals with speech-like qualities occurs with "sine-wave speech," in which the formants of natural speech are replaced by suitably amplitude- and frequency-modulated pure tones. Naive listeners to sine-wave sentences often report an initial impression of undifferentiated whistles, which can change suddenly (and usually irreversibly) to a percept with sufficient phonetic quality so that the original sentence can be reported accurately (e.g., Remez, Rubin, Pisoni, \& Carrell, 1981). Thus, in the course of Whalen and Liberman's (1987) procedure, the listeners may have become spontaneously aware that falling and rising glides had perceptual properties in common with " $\mathrm{d}$ " and " $\mathrm{g}$," respectively. To the extent that this is a possibility, it is pertinent to know not only whether glide identification is at chance at the outset of the experiment, but also to establish whether it remains so at the end of the experiment.

The duplexity threshold was determined in Whalen and Liberman's (1987) experiment by the method of adjustment. Their subjects were asked to adjust the intensity of the glide in syllabic context, apparently from below threshold until a nonspeech whistle could just be heard. Duplexity thresholds were obtained separately for " $d$ " and " $g$ " glides, and averaged, respectively, $-6.4 \mathrm{~dB}$ and $0.0 \mathrm{~dB}$ relative to the third formant steady state. The 
method of adjustment used in this way may not be the best choice of psychophysical procedure to establish the duplexity threshold, for two reasons. First, thresholds measured using the method of adjustment are susceptible to variation in the strictness of the criterion that an observer sets for detection, and a method such as twoalternative forced choice, which is less susceptible to this type of bias, may be preferable. Second, it is usually found that method-of-adjustment trials started only from below threshold tend to overestimate the threshold. When method of adjustment is used, a safer estimate of threshold is obtained if trials begin with the stimulus level set above and below threshold with equal frequency. If the procedure used by Whalen and Liberman did systematically overestimate the duplexity threshold, the possibility remains that, even in their "below duplexity threshold" condition, glides in syllabic context could have been detected as distinct perceptual entities.

This raises two questions that merit attention. First, can their data be taken to show that there is a genuinely subthreshold glide level that is nonetheless sufficient to disambiguate the syllabic base? Second, it is argued above that the glides themselves could carry schematic phonetic information. If they do so in syllabic context when detected as distinct perceptual entities (even, if it was overestimated, below the notional duplexity threshold), could identification of the base + glide combination as " $d$ " or " $g$ " depend at least in part on the perceptual characteristics of the glides themselves, and not entirely on perceptual integration of information from base and glide?

Our objective in the present experiments was, therefore, to address these issues in a replication of Whalen and Liberman's (1987) experiment, with special attention to the following points. First, we sought a bias-free estimate of the duplexity threshold (Experiments 1 and 2). Second, we tried to determine whether consonant identification was indeed possible when glide levels were below this new estimate of the duplexity threshold (Experiments 3 and 4). Third, we attempted to explore the generality of the claim that tone glides of this type are not associated reliably with " $d$ " and " $g$ "' responses (Experiments 3 and 4).

\section{EXPERIMENT 1}

The purpose of this experiment was to obtain an unbiased estimate of the duplexity threshold using a twoalternative forced-choice procedure.

\section{Method}

Stimuli

Because Whalen and Liberman (1987) did not specify in detail the stimulus parameters they used, the base syllable we used was modeled as closely as possible after that used by Mann and Liberman (1983), to which Whalen and Liberman refer. It was constructed using a programmable hardware digital synthesizer, configured as a parallel-formant terminal-analog synthesizer. An outline of the synthesizer control parameters is given in Table 1. Intermediate values were obtained by linear interpolation between the values given.

The waveforms for the tone glides were sinusoids modeled after the acoustic characteristics of third formant transitions which, when combined with the base, evoked clear percepts of "da" and "ga." The glides were computed according to the parameters outlined in Table 2, with linear interpolation between the values given.

The independent variable in the experiment was the peak spectrum level of the tone glides relative to that of the third formant steady state. The range of levels used was from -31 to $-11 \mathrm{~dB}$ for the " $\mathrm{d}$ " glide, and from -24 to $-4 \mathrm{~dB}$ for the " $\mathrm{g}$ " glide, in each case in 2-dB steps, giving 11 stimuli in each range.

The stimuli consisted of the base alone or a digital mixture of the base and one of the glides. They were recorded on cassette tape in appropriate sequences and presented to subjects binaurally via headphones at a comfortable level (approximately $75 \mathrm{~dB}$ SPL) in a quiet room.

\section{Procedure}

A trial in the experiment consisted of two intervals, in each of which a stimulus was presented. One interval contained the base alone and the other the base mixed with either a " $d$ " or a " $g$ " glide, with $500 \mathrm{msec}$ of silence between the two intervals. The subjects' task was to identify the interval that contained a chirp-like sound, guessing if necessary. No feedback was given about the accuracy of their responses, which were written on specially prepared response sheets. Six blocks of 44 trials were presented, with an intertrial interval of $3 \mathrm{sec}$ and an interblock interval of $10 \mathrm{sec}$. In each 44-trial block, each glide type ("d" or " $g$ ") appeared once at each level and once in each interval, in a pseudorandom order. Each subject, therefore, made 12 forced-choice judgments for each glide level (six blocks, two presentations per block). To familiarize subjects with the procedure, a brief sequence of 8 practice trials

Table 1

Synthesizer Control Parameters for the Syllable Base

\begin{tabular}{|c|c|c|c|c|c|c|c|}
\hline \multirow[b]{2}{*}{$\begin{array}{c}\text { Time } \\
(\mathrm{msec})\end{array}$} & \multicolumn{2}{|c|}{ First Formant } & \multicolumn{2}{|c|}{ Second Formant } & \multicolumn{2}{|c|}{ Third Formant } & \multirow{2}{*}{$\begin{array}{l}\text { Fundamenta) } \\
\text { Frequency } \\
(\mathrm{Hz})\end{array}$} \\
\hline & $\begin{array}{l}\text { Freq } \\
(\mathrm{Hz})\end{array}$ & $\begin{array}{c}\text { Ampl } \\
(\mathrm{dB})\end{array}$ & $\begin{array}{l}\text { Freq } \\
(\mathrm{Hz})\end{array}$ & $\begin{array}{c}\text { Ampl } \\
\text { (dB) }\end{array}$ & $\begin{array}{l}\text { Freq } \\
(\mathrm{Hz})\end{array}$ & $\begin{array}{l}\text { Ampl } \\
\text { (dB) }\end{array}$ & \\
\hline 0 & 279 & -5 & 1764 & -5 & & $-\infty$ & 110 \\
\hline 25 & & 0 & & 0 & & $-\infty$ & \\
\hline 50 & 765 & & 1230 & & 2527 & -3 & \\
\hline 225 & & 0 & & 0 & & -3 & \\
\hline 245 & & -8 & & -8 & & -13 & \\
\hline 250 & 765 & $-\infty$ & 1230 & $-\infty$ & 2527 & $-\infty$ & 80 \\
\hline
\end{tabular}

Note-Amplitudes are expressed relative to their maximum value. Half-power bandwidths for the first, second, and third formants were 60,80 , and $120 \mathrm{~Hz}$, respectively. 
Table 2

Frequency and Amplitude Parameters for the Tone Glides

\begin{tabular}{ccccccc}
\multicolumn{3}{c}{ "d" } & Tone Glide & & \multicolumn{3}{c}{ "g" Tone Glide } \\
\cline { 5 - 7 } $\begin{array}{c}\text { Time } \\
(\mathrm{msec})\end{array}$ & $\begin{array}{c}\text { Freq } \\
(\mathrm{Hz})\end{array}$ & $\begin{array}{c}\text { Ampl } \\
(\mathrm{dB})\end{array}$ & $\begin{array}{c}\text { Time } \\
(\mathrm{msec})\end{array}$ & $\begin{array}{c}\text { Freq } \\
(\mathrm{Hz})\end{array}$ & $\begin{array}{c}\text { Ampl } \\
(\mathrm{dB})\end{array}$ \\
\hline 0 & 3196 & -3 & 0 & 1853 & -3 \\
45 & 2527 & 0 & 45 & 2527 & 0 \\
50 & 2527 & $-\infty$ & 50 & 2527 & $-\infty$ \\
\hline
\end{tabular}

Note-Amplitudes are expressed relative to their maximum value.

preceded the experimental trials, in which the glides were presented well above threshold.

\section{Subjects}

Eight subjects were tested. None reported any hearing impairment.

\section{Results and Discussion}

The results are shown graphically in Figure 2, which plots psychometric functions separately for the " $\mathrm{d}$ " and " $g$ " glides. It is clear from these data that the duplexity thresholds estimated by any conventional criterion are lower than those obtained by Whalen and Liberman (1987) using method of adjustment, which were -6.4 and $0 \mathrm{~dB}$ for " $d$ " and " $g$," respectively. For example, in this experiment, the equivalent thresholds corresponding to $75 \%$ correct performance were approximately -19.5 and $-10.5 \mathrm{~dB}$.

There are two important caveats to acknowledge before drawing direct comparisons between the data from this experiment and those of Whalen and Liberman (1987). First, although we made explicit attempts to model Whalen and Liberman's stimuli closely, it is difficult to establish just how well we succeeded. It is possible, but in our view highly unlikely, that the differences in the duplexity thresholds derive from some subtle differences between the two sets of stimuli.

Second, and perhaps of more potential significance, the subjects' tasks were different. In Whalen and Liberman's (1987) procedure, the subjects varied the intensity of the tone glide "until the whistle was just audible." In our procedure, the subjects' task was to identify the interval containing a chirp-like sound. Our own informal listening suggested that this was a natural task, and it was our very strong impression from subjects' comments after the experiment that they were indeed doing what we asked them to do. But, even though no feedback was given, there are other response strategies that they might have adopted. Since the syllable base, when presented alone, had an ambiguous initial consonant, it is possible that in responding to the interval with base + glide, the subjects did not perceive a chirp but were actually identifying the interval that had a syllable with a clearer, less ambiguous consonant. However, a similar ambiguity of strategy is possible in Whalen and Liberman's procedure. Rather than waiting for a clearly audible whistle, their subjects could have set the glide attenuation at a level just sufficient to evoke a clearly identifiable consonantal percept. In view of this, we believe that it is appropriate to compare the duplexity thresholds between the two experiments.
The appropriateness of the comparison would, however, be reinforced by a demonstration that similar duplexity thresholds to those measured in Experiment 1 were also obtained in a procedure that attempted to control for the possibility that the thresholds in Experiment 1 were not duplexity thresholds, but simply thresholds for syllable perception. ${ }^{1}$ Experiment 2 was undertaken chronologically after all the other experiments reported here, with the objective of providing this demonstration.

\section{EXPERIMENT 2}

The purpose of Experiment 2 was to obtain and compare two estimates of the duplexity thresholds, each using a two-alternative forced-choice procedure. One estimate was obtained using the procedure of Experiment 1. For the other estimate, we sought a stimulus arrangement that would make it less likely that subjects would respond by identifying the stimulus interval containing the clearer consonant, rather than the interval containing the chirp-like sound. Accordingly, the procedure of Experiment 1 was modified so that base + glide stimuli were paired on each trial not with the base stimulus alone, but with a base stimulus having a periodic third formant transition resonance with the same frequency and peak amplitude characteristics as the tone glide. Stimulus pairs constructed in this way are approximately equal in the ambiguity of the initial consonant at the start of each syllable of the pair, but the periodicity and spectral properties common to the base and the periodic resonance transition reduce the probability that the resonance transition will be heard as a sep-

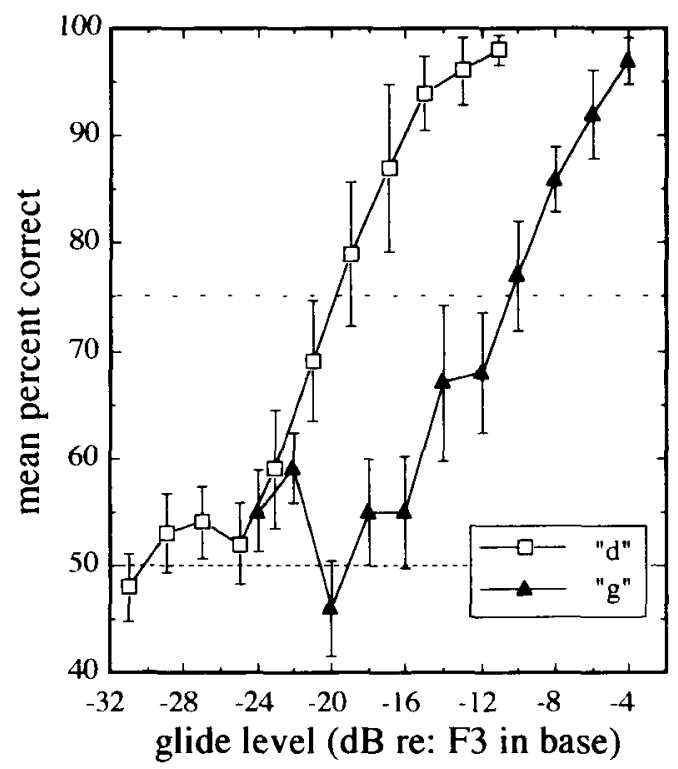

Figure 2. Chirp detection performance in Experiment 1, with " $d$ " and "g" glides in trials consisting of base versus base + glide. Units on the horizontal axis are decibels with respect to the level of the third formant steady state in the base syllable. Standard errors are shown. Dashed horizontal lines show performance at $75 \%$ correct and $50 \%$ correct (chance). 
arate perceptual chirp-like entity until levels well above those at which the chirp evoked by a pure-tone glide is clearly perceptible.

This procedure was intended to determine whether the duplexity threshold measured in Experiment 1 was actually a threshold for the perception of a syllable with an unambiguous initial consonant. We do not pretend that the procedure provides an ideal or complete control for the possibility that Experiment 1 measured some other threshold than a duplexity threshold. Although twoalternative forced choice has some advantages over method of adjustment in minimizing the influence of response bias on threshold measurements, it shares with method of adjustment the problem that the experimenter can only specify the stimulus property to which attention should be given by means of the instructions to the subject and by careful selection of practice trials. The specificity of those instructions, the simplicity of the task from the subjects' point of view, and the pattern of the data obtained are the experimenters' major insurance that the subjects are responding to the intended stimulus property.

With the experimental design used here, it was our intention to manipulate stimulus properties so as to minimize differences between the clarity of the consonants in the two intervals on a trial. Such manipulations should have affected one of the possible unintended thresholdsthat for syllable clarity-but not the intended duplexity threshold for chirp detection. If duplexity thresholds obtained with this modified procedure are substantially higher than estimates made with the original procedure, it would suggest that our original duplexity thresholds were indeed confounded with a "syllable threshold." However, if the estimates of duplexity threshold for the two procedures are similar, the likelihood is increased that the subjects in Experiment 1 were detecting the chirp as instructed and thus that the original estimates of duplexity threshold were reliable.

\section{Method}

\section{Stimuli}

The base stimulus and tone glides were the same as those used in Experiment 1. In addition to these, a set of periodic third formant transitions was created with center frequency and amplitude characteristics like those of the tone glides (Table 2) and with bandwidth and fundamental frequency characteristics like those of the steady state third formant in the base (Table 1).

In both conditions of the experiment, the independent variable was the peak spectrum level of the tone glides and resonance glides (when present) relative to that of the third formant steady state. The range of levels used was from -31 to $-11 \mathrm{~dB}$ for the " $\mathrm{d}$ " tone and resonance, and from -24 to $-4 \mathrm{~dB}$ for the " $g$ " tone and resonance, in each case in 2-dB steps, giving 11 stimuli in each range.

The stimuli consisted of the base alone, a digital mixture of the base and one of the tone glides, and a digital mixture of the base and one of the resonance glides. They were recorded on cassette tape in appropriate sequences and presented to subjects binaurally via headphones at a comfortable level (approximately $75 \mathrm{~dB}$ SPL) in a sound-attenuating room.

\section{Procedure}

Condition 1: Base versus base + tone glide. This was a replication of the procedure in Experiment 1, with the following minor difference: An additional block of 44 trials was presented, bringing the total number of blocks to seven, and the number of forced-choice judgments for each glide level to 14 (seven blocks, two presentations per block).

Condition 2: Base + resonance glide versus base + tone glide. As in Experiment 1, trials consisted of two intervals, in each of which a stimulus was presented. One interval contained the base mixed with either a " $d$ " or " $g$ " glide, and the other the base mixed with a periodic resonance of corresponding type and level, with 500 msec of silence between the two intervals. The subjects' task was, as before, to identify the interval that contained a chirp-like sound, guessing if necessary. The subjects were warned that they might hear chirp-like sounds at the beginning of the syllables in both intervals, and if this happened they were to choose the interval with the clearest chirp. As in Condition 1, seven blocks of 44 trials were presented, so that 14 judgments were made at each glide/resonance level. In other respects, the procedure was like that of Experiment 1.

\section{Subjects}

Eight new subjects were tested. None reported any hearing impairment.

\section{Results and Discussion}

The results are shown graphically in Figures 3a (Condition 1) and $3 b$ (Condition 2), each of which plot mean psychometric functions separately for the " $d$ " and " $g$ " glides.

Thresholds corresponding to $75 \%$ correct performance were computed from the mean functions by linear interpolation and are shown (with the corresponding thresholds from Experiment 1 for comparison) in Table 3. We argued previously that if the procedure of Condition 1 was actually measuring a threshold for syllable perception, thresholds in that condition would be systematically lower than those in Condition 2, in which the consonants in the two syllables in each trial were of approximately equal salience. The data show that for " $d$ " glides the threshold level is indeed lower in Condition 1 than in Condition 2 , but by a negligible amount $(1.9 \mathrm{~dB})$, and for " $\mathrm{g}$ "' glides the threshold level is actually somewhat higher in Condition $1(4.8 \mathrm{~dB})$.

It is noteworthy that there were differences between the results from Experiment 1 and from Condition 1 of Experiment 2, despite the intended similarity in procedure, particularly in the thresholds for " $d$ " glides. Part of this difference may be attributable to differences between subjects, but the relatively close correspondence of the thresholds for " $g$ "' glides argues against this. The lower threshold for " $\mathrm{d}$ " glides in Experiment 2, Condition 1 is more likely to derive from the fact that Experiment 2 was run in a sound-attenuating room with very low levels of ambient noise, whereas Experiment 1 was run in a (quiet) ordinary room. The higher levels of ambient noise in the latter environment probably provided a noise floor into which the lower level " $d$ " glides disappeared. In the 
(a)

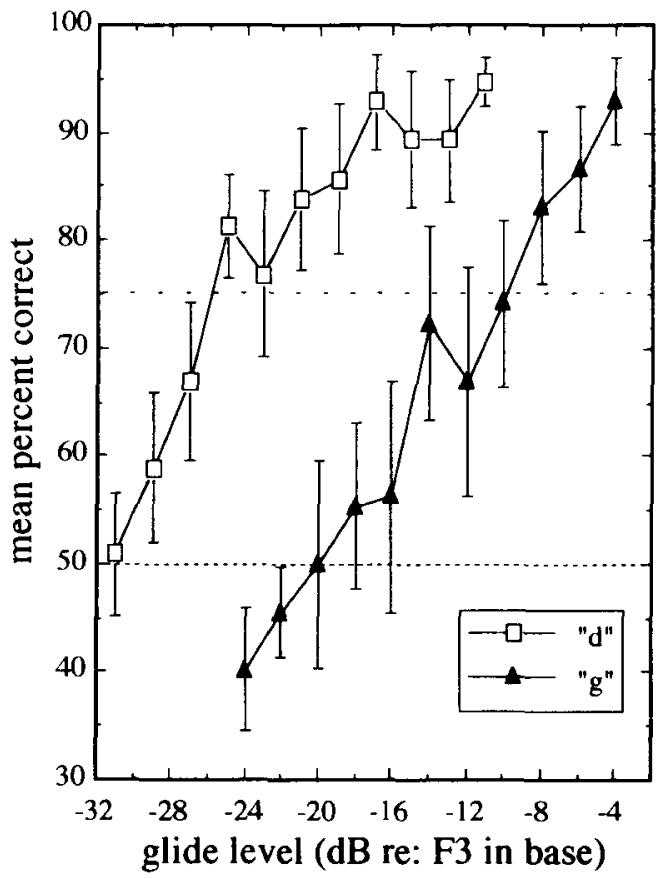

(b)

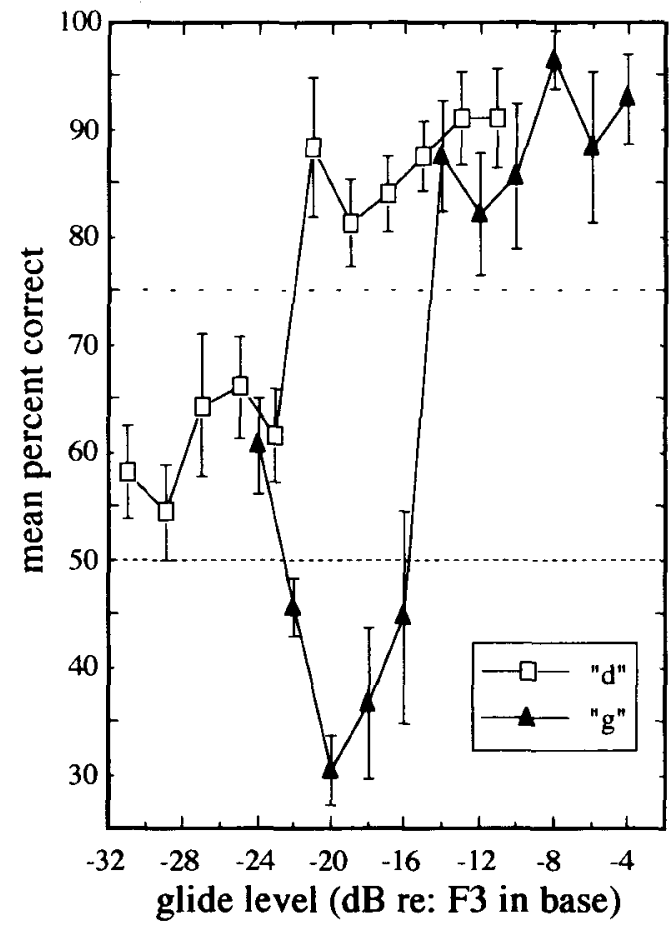

Figure 3. Chirp detection performance in Experiment 2: Condition 1, in which, as for Experiment 1, trials consisted of base versus base + tone glide (a), and Condition 2 , in which trials consisted of base + resonance glide versus base + tone glide (b). Units on the horizontal axis are decibels with respect to the level of the third formant steady state in the base syllable. Standard errors are shown. Dashed horizontal lines show performance at $\mathbf{7 5 \%}$ correct and $\mathbf{5 0 \%}$ correct (chance).
Table 3

Thresholds Corresponding to $75 \%$ Correct Mean Performance in Experiments 1 and 2

\begin{tabular}{cccc}
\hline Experiment & Condition & "d" Glide $(\mathrm{dB})$ & " $\mathrm{g}$ " Glide (dB) \\
\cline { 2 - 4 } 2 & & -19.5 & -10.5 \\
2 & 1 & -25.9 & -9.8 \\
& 2 & -24.0 & -14.6 \\
\hline
\end{tabular}

Note-In Experiment 1 and Condition 1 of Experiment 2, base + tone glide stimuli were paired with base-only stimuli. In Condition 2 of Experiment 2 , base + tone glide stimuli were paired with base + resonance glide stimuli.

low noise of the sound-attenuating room, the psychometric function is shifted to the left (compare Figures 2 and $3 a$ ), and the $75 \%$ correct threshold is correspondingly lower. A similar effect was presumably not seen for " $g$ "' glides because their higher absolute level ensured that they were above the noise floor in both experimental environments, even at their lowest level.

The broad similarity of the results from the two conditions of Experiment 2 encouraged us to believe that Experiment 1 furnished legitimate measures of duplexity thresholds that were substantially lower than those obtained by Whalen and Liberman (1987). However, our suspicion that the procedure of Condition 2 was not a flawless control was probably justified. There are several differences between the psychometric functions from Conditions 1 (Figure 3a) and 2 (Figure $3 b$ ) that suggest that the subjects may have been using more than one response strategy. For example, inspection of the " $g$ " psychometric function in Figure 3b shows a sharp decrease in mean percent correct for glide levels below $-12 \mathrm{~dB}$ to apparently systematic below-chance performance for glide levels between -16 and $-22 \mathrm{~dB}$. This suggests that the " $g$ " resonance transition could sometimes be heard out as a chirp-like entity itself, leading to systematically incorrect choice of which syllable contained the tone glide. This greater prominence of resonance glides relative to tone glides in the -16 to $-22 \mathrm{~dB}$ range may be attributable to the detection of unmasked energy as chirp-like on the high-frequency side of the center frequency through the resonance glide. As the glide energy decreased further, masking presumably extended over all the resonance glide, so that both tone and resonance glides became equally undetectable and performance on chirp detection returned to chance levels. The absence of a corresponding effect for the " $\mathrm{d}$ " function is probably due to the greater difference in mean frequency between the " $d$ " glide and the major concentrations of spectral energy in the base. Whatever the reasons for these peculiarities in the data, they serve to emphasize the challenge presented by unbiased measurement of duplexity thresholds (see Hall \& Pastore, 1992).

We consider below the relevance of the differences in duplexity thresholds in the present Experiment 1 and in Whalen and Liberman (1987) for the data from other conditions in Whalen and Liberman's experiment. In Experiment 3 we attempted to replicate those conditions, 
using duplexity thresholds estimated from the data of Experiment $1 .^{2}$

\section{EXPERIMENT 3}

Whalen and Liberman (1987) gave their subjects a sequence of four tasks. First, tone glides were presented in isolation for identification as "da" or "ga." Their expectation was that, in isolation, neither glide would sound like "da" or "ga." Second, tone glides were presented above duplex threshold in syllabic context (i.e., base + glide) in the center of a stimulus triad, the first and third members of which were isolated rising or falling glides. The subjects' task was to match the duplex chirp to the isolated glide it most resembled. Whalen and Liberman expected the percept of the isolated glide to be readily matched to that of the glide in syllabic context. Third, base + glide syllables with the glide levels at $4 \mathrm{~dB}$ below their previously measured "da" duplexity threshold were presented for identification of the consonant as "da" or "ga." Fourth, the identification task was repeated with glide levels at $6 \mathrm{~dB}$ above their duplexity threshold for "ga." Whalen and Liberman predicted that both above and below duplexity threshold the tone glide direction should determine whether the syllable is heard as " $\mathrm{da}$ " or "ga." We repeated all these conditions in the order indicated, using the estimate of the duplexity threshold from our first experiment but with different subjects, and added a fifth task that was simply a repetition of the first task-isolated tone glide identification--at the end of the experiment. This additional glide identification task was included, as discussed previously, to monitor any changes in the subjects' perception of phonetic information in the glides during the course of the experiment.

\section{Method}

\section{Stimuli}

The stimuli were those used in Experiment 1. We selected the fairly stringent criterion of $66 \%$ correct for the duplexity thresholds, roughly at the midpoint of the region of maximal slope in the psychometric functions, to minimize the possibility that consonant identification in base + glide syllables could depend on suprathreshold glide identification. The glide levels corresponding to $66 \%$ correct in Experiment 1 were -21 and $-12 \mathrm{~dB}$ relative to the third formant steady state, for " $\mathrm{d}$ " and " $\mathrm{g}$," respectively. As before, stimulus sequences were recorded on cassette tape and presented binaurally over headphones at a comfortable level (approximately $75 \mathrm{~dB}$ SPL for syllabic stimuli).

\section{Procedure}

The tasks described below were administered in the following order in a single short session. In each case, the subjects made their responses by marking a specially prepared response sheet.

Task 1: Glide identification. The subjects were presented with 20 " $\mathrm{d}$ " glides and 20 " $\mathrm{g}$ " glides in isolation in a pseudorandom order, with the instruction to identify them as " $\mathrm{d}$ " or " $\mathrm{g}$." The same order was used for each subject. Both types of glide were presented at the same clearly audible level, $6 \mathrm{~dB}$ above the duplexity threshold for " $g$ " ( $-6 \mathrm{~dB}$ re F3 steady state; Whalen \& Liberman, 1987, did not specify the stimulus level they used in this task). The interstimulus interval was $3 \mathrm{sec}$.
Task 2: Glide matching. Stimulus triads were prepared in which the central item in the triad was a base + glide stimulus and the first and last items were an isolated " $d$ " glide and an isolated " $g$ " glide or, equally frequently, vice versa. In the base + glide combination, there were 20 occurrences of "d" glides and 20 occurrences of " $g$ " glides in a pseudorandom order. Both types of glide were presented at the same level, $6 \mathrm{~dB}$ above the duplexity threshold for " $g$ '" ( $-6 \mathrm{~dB}$ re F3 steady state; Whalen \& Liberman, 1987, used $+6 \mathrm{~dB}$ ). The task was an AXB matching task, in which the subjects had to indicate to which isolated glide (i.e., first [A] or second [B]) the glide in the base + glide combination was most similar. The interstimulus interval within a triad was $500 \mathrm{msec}$, and the intertrial interval was $4 \mathrm{sec}$.

Task 3: Consonant identification below duplexity threshold. The subjects were presented with a pseudorandom sequence of 40 base + glide stimuli, of which 20 had " $d$ " glides and 20 had " $g$ ", glides. The glide level for both types of glide was $6 \mathrm{~dB}$ below the "d" duplexity threshold ( $-27 \mathrm{~dB}$ re F3 steady state; Whalen \& Liberman, 1987, used $-10.4 \mathrm{~dB}$ ). The choice of $6 \mathrm{~dB}$ below the duplexity threshold rather than the $4 \mathrm{~dB}$ used by Whalen and Liberman was motivated by our wish for a glide level that gave unambiguous chance performance, as determined in the chirp detection task of Experiment 1. The subjects' task was to identify the initial consonant of the syllable as " $d$ " or " $g$." The interstimulus interval was $3 \mathrm{sec}$.

Task 4: Consonant identification above duplexity threshold. The format of this condition was similar to Task 3 , but with the glide level set to $6 \mathrm{~dB}$ above the duplexity threshold for " $g$ " ( $-6 \mathrm{~dB}$ re F3 steady state; Whalen \& Liberman, 1987 , used $+6 \mathrm{~dB}$ ). This level gave over $90 \%$ correct chirp detection performance in Experiment 1.

Task 5: Glide identification. This was a repetition of Task 1.

\section{Subjects}

Eight new subjects were tested. None reported any hearing impairment.

\section{Results and Discussion}

The results for each task expressed in terms of the overall percentage of correct responses are shown in Table 4, and graphically in Figure 4. Although there are several differences between their stimuli and ours, for the purposes of comparison, the corresponding data from Whalen and Liberman's (1987) experiment are also shown in

Table 4

Results from Experiment 3, Expressed as Mean Percent Correct for Each Task

\begin{tabular}{lccccc}
\hline Subject & Task 1 & Task 2 & Task 3 & Task 4 & Task 5 \\
\hline 1 & 97.5 & 70.0 & 42.5 & 97.5 & 100.0 \\
2 & 50.0 & 27.5 & 55.0 & 67.5 & 85.0 \\
3 & 90.0 & 57.5 & 50.0 & 82.5 & 82.5 \\
4 & 100.0 & 95.0 & 65.0 & 97.5 & 95.0 \\
5 & 17.5 & 55.0 & 47.5 & 95.0 & 100.0 \\
6 & 100.0 & 42.5 & 82.5 & 97.5 & 77.5 \\
7 & 95.0 & 55.0 & 47.5 & 92.5 & 72.5 \\
8 & 0.0 & 45.0 & 55.0 & 65.0 & 0.0 \\
$M$ & & & & & \\
$S E M$ & 68.8 & 55.9 & 55.6 & 86.9 & 76.6 \\
$t(7)$ & 14.4 & 7.1 & 4.5 & 4.8 & 11.5 \\
$p$ & 1.30 & 0.83 & 1.24 & 7.63 & 2.31 \\
\hline
\end{tabular}

Note-Tasks 1 and 5: glide identification; Task 2: glide matching; Task 3: consonant identification below duplexity threshold; Task 4: consonant identification above duplexity threshold. See text for details of table entries and tasks. 


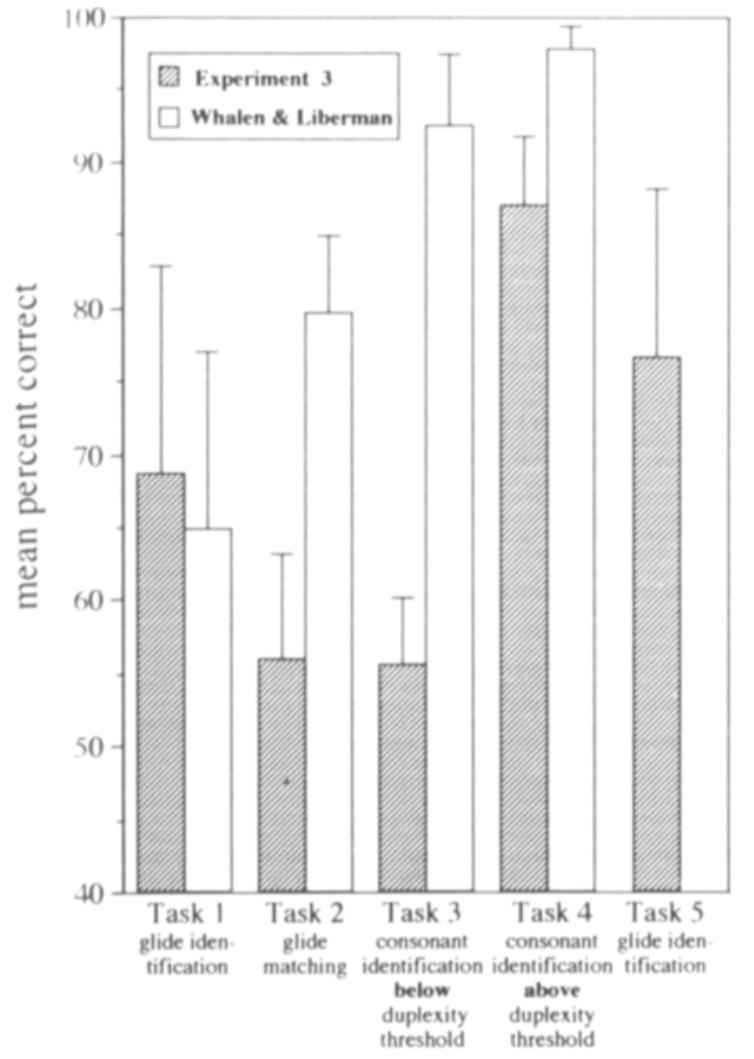

Experimental conditions

Figure 4. Mean percent correct for the present Experiment 3 and for Whalen and Liberman (1987). Standard errors are shown above each bar. See text for details of tasks.

Figure 4. Table 4 also shows, for each task, the mean percentage correct $(M)$, the standard error of the mean (SEM), the value of Student's $t$, computed to compare the obtained mean with chance performance $(50 \%)$, and the probability associated with that value of $t$. Responses to stimuli with " $d$ " and "g" glides are collapsed together. Although there was a tendency for " $d$ " glides at a given level to be recognized more accurately than " $g$ " glides at the same level, the difference in performance was not significant, and the pattern of data was similar for both glide types.

We consider first the possibility that the glides themselves can carry information sufficient for reliable consonant identification. Initially, this appears not to be the case overall, as mean glide identification performance is not reliably different from chance in Task 1 at the start of the testing session. This is the result obtained by Whalen and Liberman (1987). However, scrutiny of the data of individual subjects shows that, as in their study, some subjects do adopt a reliable and correct response strategy from the outset-5 of the 8 subjects here scored $90 \%$ correct or better.

As we have already acknowledged, the adoption of a correct response strategy does not demonstrate sensitivity to any phonetic information carried by the glides; it requires only an ability to discriminate the glides reliably and an arbitrary alignment of " $d$ " response with falling glides and " $g$ " responses with rising glides. However, as in Whalen and Liberman's (1987) experiment, the mean percentage correct is greater than $50 \%$, as would be expected if the assignment of responses to glides was not completely arbitrary. We do not suggest that falling and rising glides sound like " $\mathrm{d}$ " and " $\mathrm{g}$," but simply that the glides may have perceptual characteristics sufficiently similar to third formant transitions in alveolar and velar consonant-vowel syllables as to make the initial assignment of labels to glides not wholly random.

In the repetition of the glide identification task at the end of the experiment (Task 5), the percentage of correct responses $(76.6 \%)$ was greater than at the beginning (Task 1), and was reliably better than chance performance. However, the difference in performance between Tasks 1 and 5 was not statistically significant. Nonetheless, the finding that average performance increases slightly (and some individual subjects' performance markedly-see Subjects 2 and 5 in Table 4) gives tentative credence to the possibility that exposure to the glides, perhaps particularly in syllabic context, can lead to an increase in the use of any phonetic information they may carry. It will be of significance in the present context if subjects became aware of phonetic information in the glides before or during tasks that involved consonant identification in base + glide syllables (Tasks 3 and 4), given the possibility that consonant identification could have been achieved on the basis of glide characteristics alone.

If the information that allows subjects to derive consonant identity from the glides alone is also available when the glides are above duplexity threshold in syllabic context, consonant identification in syllables consisting of base + glide could depend in part on information in the glide itself, and not require integration of the base and glide elements of the stimulus ensemble. In this context, it is perhaps noteworthy that Subject 8 (Table 4), who chose to label falling glides as " $\mathrm{g}$ " and rising glides as "d," was least successful at consonant identification in Task 4. However, successful consonant identification in base + glide stimuli by use of information from the glide alone requires that subjects can discriminate the glides in syllabic context. If rising and falling glides in syllabic context cannot be discriminated reliably, the capacity of glides to disambiguate the initial consonant in the syllable base must derive from an integration of information from base and glide (see also Pastore, Li, \& Layer, 1990).

A measure of the subjects' ability to discriminate rising and falling glides in syllabic context (though not necessarily a good one-see below) is provided by Task 2 . This task involved matching isolated glides at a level above the duplexity threshold with similar glides in base + glide stimuli. Whalen and Liberman's (1987) subjects performed significantly above chance on this task, and we had expected our subjects to do the same, but as Table 4 and Figure 4 show, they did not. Whalen and Liberman comment that glide matching accuracy did depend on glide 
level and refer in their note 7 on page 171 to a replication of Task 2 with glide level $-10.4 \mathrm{~dB}$ re F3 steady state that gave chance performance. Thus, the difference in outcome on Task 2 may be the result of our choice of such a strict criterion for the duplexity threshold. Although the glides in Task 2 were clearly detectable, they may have been below a "discrimination threshold" at which they could reliably be told apart and thus successfully matched.

The final notable difference between Whalen and Liberman's (1987) results and ours is in the results from Task 3--consonant identification below duplexity threshold. In Task 3, when glide level was below Whalen and Liberman's duplexity threshold, their subjects could reliably identify the consonant in base + glide syllables. With the duplexity threshold defined in the stringent way we have done, on the basis of data from Experiment 1, our subjects gave chance performance on Task 3 . We take our results to indicate a need for caution in accepting Whalen and Liberman's claim that there is a range of subthreshold glide levels that can influence consonant identity in base + glide syllables. Our data suggest that this may have arisen in their experiment because of the tendency for their threshold determination procedure to overestimate the duplexity threshold.

The reliably above-chance performance on Task 4consonant identification above duplexity thresholdechoed that obtained by Whalen and Liberman (1987), although the absolute levels of performance were slightly lower in our data. This confirmed that information from glides in base + glide syllables can serve to disambiguate the initial consonant when the glide level is above threshold. ${ }^{3}$

The differences in outcome between the two studies were sufficiently marked to motivate a more exact replication of the original (1987) Whalen and Liberman experiment. Accordingly, we repeated the experiment using glide levels that were similar to those used by Whalen and Liberman.

\section{EXPERIMENT 4}

Experiment 4 was as close a replication of the conditions used by Whalen and Liberman (1987) as we could contrive. We retained the repetition of the glide identification task (Task 1) at the end of the testing session (Task 5) as in Experiment 3, to monitor any changes in subjects' glide labeling performance during the course of the experiment.

\section{Method}

\section{Stimuli and Procedure}

These were as described for Experiment 3, except that the glide levels were as follows.

For Tasks 1,2, 4, and 5 (glide identification, glide matching, and consonant identification above duplexity threshold), glide levels were $+6 \mathrm{~dB}$ relative to the level of the third formant steady state, which was $18 \mathrm{~dB}$ above our duplexity threshold for " $\mathrm{g}$."

For Task 3 (consonant identification below duplexity threshold), glide levels were $-10 \mathrm{~dB}$ relative to the level of the third formant steady state, which was $2 \mathrm{~dB}$ above our duplexity threshold for " $\mathrm{g}$."

\section{Subjects}

Eight new subjects were tested. None reported any hearing impairment.

\section{Results and Discussion}

The results expressed as the overall mean percentage correct for each task are shown in Table 5 and graphically in Figure 5 to facilitate comparison with the results from Experiment 3 and Whalen and Liberman (1987). Re-

Table 5

Results from Experiment 4, Expressed as Mean Percent Correct for Each Task

\begin{tabular}{lccccc}
\hline Subject & Task 1 & Task 2 & Task 3 & Task 4 & Task 5 \\
\hline 1 & 100.0 & 40.0 & 92.5 & 95.0 & 100.0 \\
2 & 100.0 & 75.0 & 52.5 & 92.5 & 100.0 \\
3 & 100.0 & 15.0 & 47.5 & 92.5 & 100.0 \\
4 & 52.5 & 47.5 & 92.5 & 90.0 & 47.5 \\
5 & 47.5 & 45.0 & 72.5 & 100.0 & 5.0 \\
6 & 100.0 & 50.0 & 75.0 & 72.5 & 100.0 \\
7 & 100.0 & 62.5 & 67.5 & 100.0 & 100.0 \\
8 & 92.5 & 60.0 & 100.0 & 100.0 & 77.5 \\
$M$ & & & & & \\
$S E M$ & 86.6 & 49.4 & 75.0 & 92.8 & 78.8 \\
$t(7)$ & 8.04 & 6.32 & 6.76 & 3.22 & 12.45 \\
$p$ & 4.54 & -0.10 & 3.70 & 13.30 & 2.31 \\
\hline
\end{tabular}

Note-Tasks 1 and 5: glide identification; Task 2: glide matching; Task 3: consonant identification below duplexity threshold; Task 4: consonant identification above duplexity threshold. See text for details of table entries and tasks.

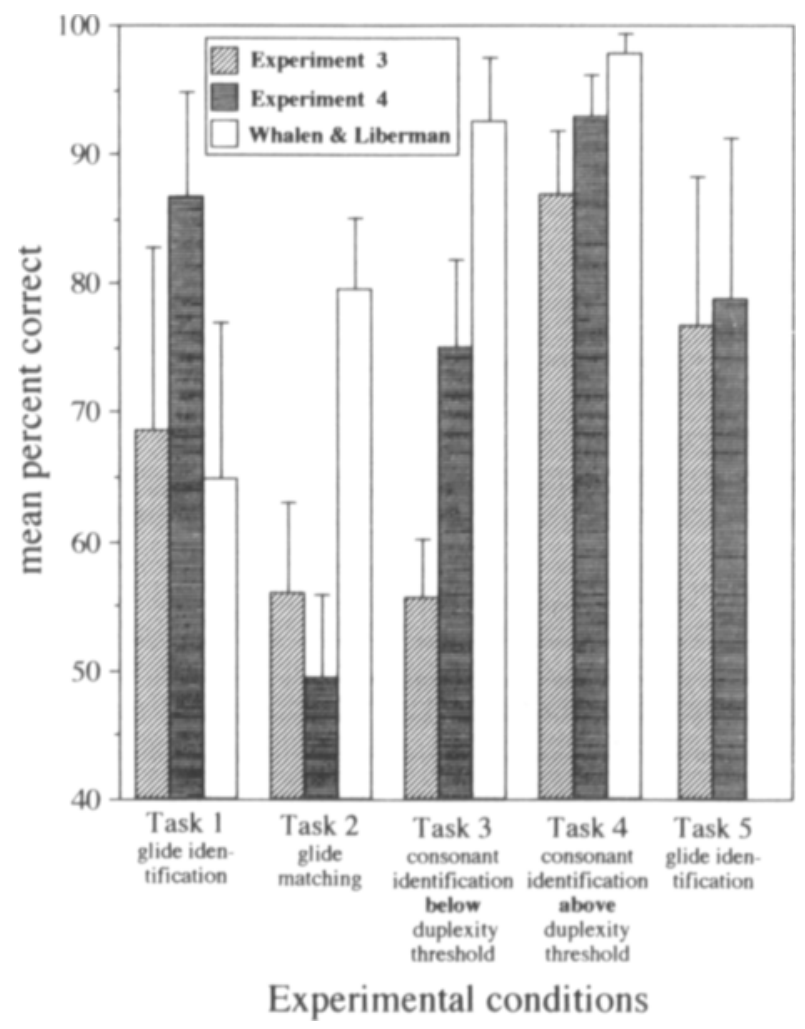

Figure 5. Mean percent correct for the present Experiments 3 and 4 and for Whalen and Liberman (1987). Standard errors are shown above each bar. See text for details of tasks. 
sponses to stimuli with " $\mathrm{d}$ " and "g" glides are again collapsed together. As before, there was a tendency for " $d$ " glides at a given level to be recognized more accurately than " $g$ " glides at the same level, but the difference in performance was not significant, and the pattern of data was similar for both glide types.

It is clear that the increase in glide level influenced the results, as might have been expected. Consonant identification "below" duplexity threshold-Task 3-was more accurate than in Experiment 3, and was reliably above chance. Whalen and Liberman (1987) also obtained this outcome, and interpreted it as indicating that the glides at this level were in a "preemptiveness interval," where they were undetectable as chirps but could still determine the identity of the syllabic consonant. We predict the result on the basis of the data from Experiment 1, without resort to the notion of preemptiveness, as the glide levels were above our measures of duplexity threshold and were therefore of themselves potentially capable of providing phonetically relevant information about consonant identity. The finding that consonant identification in Task 4consonant identification above duplexity threshold-was reliably above chance was also to be expected, as the glide levels were 25.5 and $18 \mathrm{~dB}$ above our thresholds for falling and rising glides, respectively. However, despite our explicit attempt to replicate Whalen and Liberman's procedure and stimulus characteristics, clear differences remained between these results and those found by Whalen and Liberman.

First, even at the outset of the testing session, the majority of the subjects in Experiment 4 were able to make reliable and correct identifications of the isolated glides as " $d$ "' or " $g$." Performance overall was significantly above chance for both Task 1 and its repetition at the end of the experiment (Task 5), and 5 of the 8 subjects gave perfect performance on both tasks. This suggests that some schematic but distinctive phonetic information is available in isolated glides. The absolute level of performance on this task decreased slightly during the experiment, but, as in Experiment 3, the difference between performance on Tasks 1 and 5 was not significant. Thus, there is no evidence to support the hypothesis that exposure to glides during the experiment increased the salience of any phonetic information they may carry.

Second, as we found in Experiment 3, performance on the glide matching task (Task 2) was not better than chance. This result stands in contrast to the significantly above-chance accuracy with which glides were matched in Whalen and Liberman's (1987) experiment. However, our result appears to be reliable, since we have obtained the same outcome in both Experiments 3 and 4 at glide levels that were, in both cases, well above threshold. Chance performance on the glide matching task suggests that the chirp percept evoked by suprathreshold glides presented in the context of other acoustic energy (in this case, the syllable base) is not equivalent to the percept evoked by suprathreshold glides presented in isolation.
In the discussion of Experiment 3, we raised the question of whether Task 2 provided a good measure of glide discriminability. Certainly the apparent inconsistency between above-chance identification of isolated glides (Task 1) and poor matching of glides in syllabic context (Task 2) deserves some comment. To score above chance in Task 1 , listeners must focus on the inherent properties of the glides, since they are presented in isolation. Task 2 may have been made more difficult by the fact that the procedure involved presentation of a triad of sounds including both glide types. This may have served to emphasize their common properties for the subject; specifically, their identical offset frequencies. It is well established that pitch matches to tone glides tend to be made near the final frequency of the glide (e.g., Nábelek, Nábelek, \& Hirsch, 1970), a tendency which, in this case, would increase the perceived similarity of the rising and falling isolated glides and render the matching task correspondingly more difficult. Thus, the procedure of Task 2 may not be an ideal way to establish the perceptual similarity of isolated glides and chirps in base + glide syllables.

There are, in any case, psychoacoustical grounds for suspecting that the auditory representations of glides presented with the base syllable may not be equivalent to those evoked by glides presented in isolation. There are at least two ways in which the stimulus context provided by the base might affect the auditory representation of the glides. First, there is a well-established tendency (e.g., Bailey, 1987; Egan \& Hake, 1950) for masking to spread upward in frequency from simultaneous regions of relatively intense lower frequency energy. The presence of the first and second formant transitions in the first $50 \mathrm{msec}$ of the base during the glide might thus be expected to have some simultaneous masking effect on glides in syllabic context, which will not arise for isolated glides. Second, there is a weak and shortlived tendency for masking to occur backward in time from high-energy stimulus regions (e.g., Elliott, 1971; see also Bentin \& Mann, 1990). Although a weak effect, it may nonetheless be a potential basis for some degradation of the auditory representation of the final portion of glides in syllabic context by the immediately following third formant steady state in the base.

Some reduction in precision may thus be expected in the auditory representation of a glide when it is presented with the base, compared with its representation when presented in isolation. This is consistent with our own experience when listening informally to base + glide stimuli-although a chirp will be audible above its detection threshold, the glide level must be increased further before one can say reliably whether the glide is rising or falling. This impression of a difference between detection and discrimination thresholds is much weaker when listening to a range of levels of glides in isolation. Evidently, these effects of context on the auditory representation of the glide must be limited, as the results of Tasks 3 and 4 show that information from the glides can serve 
to disambiguate the consonant in base + glide syllables. However, the context effects that do occur are likely to influence performance particularly on a matching task like Task 2, involving the judged similarity of glides in isolation and in syllabic context.

All this leaves unexplained the difference between our result on Task 2 and that obtained by Whalen and Liberman (1987). We doubt that the difference in outcome should be attributed to any differences between our stimuli and theirs, which, at worst, are likely to be minor and irrelevant to the demands of the glide matching task. We argued previously that the structure of the trials in this task may predispose attention to the perceptual similarities between the glide types, and it is possible that the task was more difficult for our subjects because, for reasons unknown, they focused more on glide similarities than did the subjects in Whalen and Liberman's experiment.

Given our suspicion that Task 2 may not be an optimal measure of the discriminability of glides in syllabic context, and the clear demonstrations from Tasks 1 and 5 that rising and falling isolated glides are discriminable and can be identified reliably with " $d$ " and " $g$ " responses, the possibility remains that identification of base + glide stimuli could, in principle, be based on glide identity alone, and not integration of information from base and glide. However, in practice, both glide identity and integration of information from base and glide are likely to play a role in consonant identification.

\section{GENERAL DISCUSSION}

Our initial objectives in undertaking these experiments were, first, to improve on the method of estimating the duplexity threshold used by Whalen and Liberman (1987), and, second, to explore the possibility that information from tone glides alone can contribute to consonant identity in base + glide syllables. Experiments 1 and 2 addressed the first objective, and yielded estimates of the duplexity threshold that were considerably lower than those reported by Whalen and Liberman. Experiments 3 and 4 addressed the second objective, and showed that rising and falling tone glides could be labeled reliably as " $g$ " and "d."

Our estimates of the duplexity thresholds, taken with the data from Experiments 3 and 4, suggest that glides below the duplexity threshold provide no perceptual information. That is, there were no glide levels that did not evoke chirp percepts but did reliably disambiguate the consonant in the base syllable. Whalen and Liberman (1987) reported that there were glide levels below their duplexity threshold that were nonetheless capable of influencing consonant identity in base + glide syllables. They took that result to indicate the operation of a phonetic module that preempts the phonetically relevant parts of the signal. Our results suggest that this "preemptiveness interval" should be attributed to a tendency for their procedure to have overestimated the duplexity threshold. Our data do not exclude the possibility that in some demonstrations of duplex perception, subthreshold levels of the discriminative stimulus component may influence perception of the complete stimulus ensemble (see, e.g., Hall \& Pastore, 1992). However, this is not seen convincingly in the duplex perception demonstrated in the stimuli we have used here.

The performance of our subjects when labeling isolated glides suggested that reliable judgments of consonant identity in base + glide syllables might be made on the basis of information from the glides alone, without involving the integration of information from the glide and the base syllable. To the extent that this is true, it implies that phonetic labeling of base + glide syllables need not require that "sinusoidal transitions combine with the formantsynthesised base to make coherent phonetic percepts" (Whalen \& Liberman, 1987, p. 170). However, in identifying consonants in base + glide syllables, subjects are likely to make use of any and all of the information that is at their disposal, and our finding that isolated glides may carry phonetically relevant information certainly does not rule out the use of information derived from perceptual integration of base and glide. Indeed, the finding in Experiments 3 and 4 that suprathreshold glides in base + glide syllables could not be matched reliably with isolated glides, suggesting that the percepts evoked by glides in isolation were not equivalent to those evoked by glides in base + glide syllables, may be a reflection of this integrative perceptual processing.

Our explicit attempt to replicate the results of Whalen and Liberman (1987) in Experiment 4 was not wholly successful. In two of the four tasks, our subjects' data differed markedly from theirs. Our subjects could reliably assign " $d$ " and " $g$ " responses to falling and rising tone glides (Task 1), whereas theirs could not, but our subjects could not reliably match the chirp in a base + glide stimulus with an isolated rising or falling tone glide (Task 2), whereas theirs could. One of the reviewers proposed that the differences could be associated with the different linguistic experience of British and North American listeners, but we doubt whether such differences between listeners would be relevant to the tasks. We have no compelling explanations for the differences in outcome, but offer the following observations.

In the case of the glide labeling task, the marked differences in labeling strategies used by different subjects within the subject groups may provide a partial explanation. As previously discussed, an initially arbitrary, but subsequently consistent, assignment of labels to glides should yield either $100 \%$ correct or $0 \%$ correct on the task. Inconsistent labeling of glides should give 50\% correct. Although both strategies are evident in the behavior of both our and Whalen and Liberman's (1987) subjects, the majority adopted the former strategy, with only a few subjects obtaining scores around $50 \%$ correct. Of the subjects who were consistent in their labeling, overall more than half scored at least $90 \%$ correct. The argument that the glides might carry phonetically relevant information follows from the fact that, in all cases, mean performance 
was above (rather than below) $50 \%$, and reliably so in Experiment 4 (and at the end of Experiment 3). It seems possible that, given the relatively small numbers of subjects tested ( 8 in each of our experiments, 10 by Whalen and Liberman), the difference in outcome on the glide labeling task between the experiments should be attributed to chance variation in sampling of subjects from the population.

Whalen and Liberman (1987) included the glide matching task as a means to establish that the chirp percept in a base + glide syllable was comparable to the percept evoked by an isolated glide. We have argued, in the discussion of Experiment 4, that there are psychoacoustical grounds for expecting that the percepts evoked by isolated glides and by glides embedded in a base syllable might not be equivalent, and that in any case the triadic comparison procedure may not be an ideal method to demonstrate perceptual equivalence. However, as we have also noted, these observations do not provide an explanation for the differences in outcome for the supposedly similar procedures and stimuli of the glide matching task in Whalen and Liberman and Experiment 4. It is possible that there were subtle differences in the instructions to the subjects, despite the explicit use in our instructions of the words used by Whalen and Liberman to characterize the task. This discrepancy is unlikely to be resolved without further experimentation.

Notwithstanding the marked differences between our data and those of Whalen and Liberman (1987), there are several common features. When the intensity of a glide in a syllabic base + glide stimulus is varied over an appropriate range, there is variation in the salience of the "chirp" percept that the glide evokes. There is corresponding variation in the ability of the glide to disambiguate the identity of the consonant in the base syllable. On the basis of the data from their experiment, Whalen and Liberman have argued (1) that the disambiguating power of the glide derives from its integration with the base to form an articulatorily plausible auditory pattern that is perceived by a phonetic module, and (2) that duplex perceptions - the simultaneous percept of chirp and unambiguous consonant-" "support the hypothesis that the phonetic mode takes precedence in processing the transitions, using them for its special linguistic purposes until, having appropriated its share, it passes on the remainder to be perceived by the nonspeech system as auditory whistles" (p. 171).

We have considerable sympathy with the first of these claims, although we have argued above that our data suggest that integration with the base may not be the only source of the glides' disambiguating power. However, in the light of our own results, we doubt whether the second claim is justified on the basis of the data reported by Whalen and Liberman (1987).

It is not our objective here to enter into the theoretical debate concerning the special status or modularity of phonetic perception. We do not doubt the profound biological significance of speech, but it seems still to be a matter of theoretical preference whether demonstrations of duplex perception should be taken to reveal the action of a specialized phonetic module. For the moment, we prefer to believe that the pattern of the data does not force this conclusion. It is consistent with generally established properties of perception, according to which perceptual systems use any information at their disposal in an attempt to rationalize a sensory ensemble in terms of plausible events in the (in this case, auditory) world. As Woodworth (1947) observed, the reinforcement of perception is the achievement of clarity. We are comfortable with the notion that clarity in the perception of speech requires apprehension of the coherent acoustical consequences of articulation, but doubt whether the phenomenon of duplex perception has so far furnished much insight into what this process actually involves.

\section{REFERENCES}

BAILEY, P. J. (1987). Difference limens for spectral peak frequency in vowel-like stimuli. Proceedings of the XIth International Congress of Phonetic Sciences, 5, 82.4.

BENTIN, S., \& MANN, V. (1990). Masking and stimulus intensity effects on duplex perception: A confirmation of the dissociation between speech and non-speech modes. Journal of the Acoustical Society of America, 88, 64-74.

Bregman, A. S. (1987). The meaning of duplex perception: Sounds as transparent objects. In M. E. H. Schouten (Ed.), The psychophysics of speech perception (pp. 95-111). Dordrecht: Martinus Nijhoff.

Crocca, V., Bregman, A. S. (1989). The effects of auditory streaming on duplex perception. Perception \& Psychophysics, 46, 39-48.

EGAN, J. P., \& HAKE, H. W. (1950). On the masking patterns of a simple auditory stimulus. Journal of the Acoustical Society of America, 22, 622-630.

ELLIOTT, L. L. (1971). Backward and forward masking. Audiology, $10,65-76$.

Fowler, C. A., \& Rosenblum, L. D. (1990). Duplex perception: A comparison of monosyllables and slamming doors. Journal of Experimental Psychology: Human Perception \& Performance, 16, $742-754$.

Hall, M. D., \& Pastore, R. E. (1992). Musical duplex perception: Perception of figurally good chords with subliminal distinguishing notes. Joumal of Experimental Psychology: Human Perception \& Performance, 18, 752-762.

Liberman, A. M., \& Mattingly, I. G. (1989). A specialization for speech perception. Science, 243, 489-494.

Mann, V. A., \& Liberman, A. M. (1983). Some differences between phonetic and auditory modes of perception. Cognition, 14, 211-235.

Nábelek, I. V., Nábelek, A. K., \& Hirsh, I. J. (1970). Pitch of tone bursts of changing frequency. Joumal of the Acoustical Society of America, 48, 536-553.

Nusbaum, H. C. (1984). Possible mechanisms of duplex perception: "chirp" identification versus dichotic fusion. Perception \& Psychophysics, 35, 94-101.

Nusbaum, H. C., Schwab, E. C., \& Sawusch, J. R. (1983). The role of "chirp" identification in duplex perception. Perception \& Psychophysics, 33, 323-332.

Pastore, R. E., LI, X-F., \& LAYER, J. K. (1990). Categorical perception of nonspeech chirps and bleats. Perception \& Psychophysics, 48, 151-156.

Pastore, R. E., Schmuckler, M. A., Rosenblum, L., \& Szczesiul, R. (1983). Duplex perception with musical stimuli. Perception \& Psychophysics, 33, 469-474.

RAND, T. C. (1974). Dichotic release from masking for speech. Journal of the Acoustical Society of America, 55, 678-680.

Remez, R., Rubin, P., Pisoni, D., \& Carrell, T. (1981). Speech perception without traditional speech cues. Science, 212, 947-950. 
REPP, B. R. (1984). Against a role of "chirp" identification in duplex perception. Perception \& Psychophysics, 35, 89-93.

Whalen, D. H., \& Liberman, A. M. (1987). Speech perception takes precedence over non-speech perception. Science, 273, 169-171.

WOODWORTH, R. S. (1947). Reinforcement of perception. American Journal of Psychology, 60, 119-124.

\section{NOTES}

1. We were encouraged into this second measurement of duplexity thresholds by the insightful comments of Dick Pastore and Valter Ciocca, two of the reviewers.

2. We have argued that the differences in duplexity thresholds between Experiment 1 and Experiment 2, Condition 1 were probably largely attributable to differences in the ambient noise levels in the two experimental environments. The use in Experiments 3 and 4 of duplexity thresholds estimated from Experiment 1 (rather than Experiment 2) is justified by the fact that Experiments 1, 3, and 4 were run in similar environments (a quiet room) with similar levels of ambient noise, whereas Experiment 2 was run in a sound-attenuating room.

3 . We note that the glide level in this task was the same as that for Task 2-glide matching-which most of our subjects could not do. To the extent that Task 2 is a test of glide discrimination ability (an issue raised elsewhere in the paper), the result suggests that glides in syllabic context cannot be discriminated. If glide identification requires glide discrimination, the data from Tasks 2 and 4 together would suggest that consonant identification in base + glide syllables is not, in fact, very dependent on schematic phonetic information from the glide itself, but is based primarily on integration of phonetically relevant information from base and glide together. Perhaps the most plausible expectation is that listeners will use whatever information is available to them, which, in these stimuli, may derive in part from the glides themselves, and in part from the integration of the glide with the base syllable.

(Manuscript received November 20, 1991; revision accepted for publication December 8, 1992.) 